\title{
Co-simulation for the Power Generation Characteristics of an Electromagnetic Linear Actuator used in Active Suspensions
}

\author{
Anqi Peng, YanShi and Wenchao Yue \\ School of Mechanical Engineering, Nanjing University of Science and Technology, Nanjing 210094, China
}

\begin{abstract}
Compared to passive suspensions, active suspensions with electromagnetic linear actuator (EMLA) not only has higher performance in active force control, but also can regenerate energy form road disturbances. In this study, the characteristics of a new designed EMLA were investigated under generator mode by precisely co-simulating the quarter active suspension with single degree of freedom. This model, which consists of sprung mass, EMLA imported from Maxwell and a spring in parallel with the EMLA, is built in Simplorer. Simulation results show that the mechanical power and electric power of the EMLA are both proportional to the frequency and amplitude of excitation. The efficiency, defined as the ratio of electric energy to mechanical energy, decreases with the increase of frequency while nonlinearly changes with the increase of stroke. The yielded maximal efficiency of $94 \%$ occur sat the frequency of 2 Hzand the stroke ranges of $6 \mathrm{~mm}$ to $9 \mathrm{~mm}$.
\end{abstract}

\section{Introduction}

Under the road input, the passive suspensions absorb road vibration by converting kinetic energy into heat and dissipating it, but for the electromagnetic active suspensions, the vibration energy can be harvested into electricity and then stored in batteries. Meanwhile, due to the ability of variable active force, the vibration damping can be adjusted to adapt to the variable road input. So, electromagnetic active suspensions increasingly become attractive replacements for the currently installed passive suspensions, especially for the electric vehicles in which the high voltage batteries can be used to drive the linear actuator and store the electricity [1-3].

As the key component of the electromagnetic active suspensions, electromagnetic linear actuator (EMLA) operates in two modes, namely motor mode and generator mode. In motor mode, batteries deliver energy to EMLA to achieve desirable active force. In generator mode, EMLA feed energy by converting kinetic energy into electrical energy and charging batteries. In this paper, a newly designed EMLA is proposed to investigate the characteristics in generator mode. When the EMLA is used in the active suspension, the whole system, which involves electric, magnetic, and mechanic subsystems, is complicated. Therefore, co-simulation between Maxwell and Simplorer is adopted to model the $1 / 4$ electromagnetic active suspension and analyze the power generation characteristic of the EMLA under different excitations.

\section{$2 \quad 1 / 4$ Electromagnetic active suspension}

\subsection{Electromagnetic linear actuator}

In the electromagnetic active suspensions, the EMLA is a replacement for the conventional damper, so its electromagnetic force should meet the demand of vibration damping. Therefore, as shown in Fig.1, a newly designed EMLA and its test bed are proposed, its performance parameters are listed in Table 1. The proposed EMLA is a single-phase tubular device, mainly composed of inner yoke, outer yoke, and winding coils (Fig.2). The moving part is formed by permanent magnets and inner yoke. By employing quasi-Halbach array, the permanent magnets consist of three ring magnets, of which permanent magnet 1,3 are radially magnetized and magnet 2 is axially magnetized. Compared with a type of motor topology which has a stationary inner yoke, this structure just has one air-gap between the mover and the stator, which provides a closed return path for the flux and reduces the change of magnetic circuit caused by the mover's high frequency reciprocating motion. Meanwhile it also can greatly reduce the eddy current loss caused by the air-gap. The fixed portion consists of outer yoke and two coils in series. The inner and outer yokes are both made of DT4C, which has high magnetic permeability and small reluctance. The permanent magnets are made of $\mathrm{NdFe} 45 \mathrm{SH}$. The EMLA has twooperating modes, one is motor, and the other is generator. When the mover moves 
with surrounding vibration, the winding coils flux linkage is modulated by the linear movement of the mover, and the induced EMF is generated.

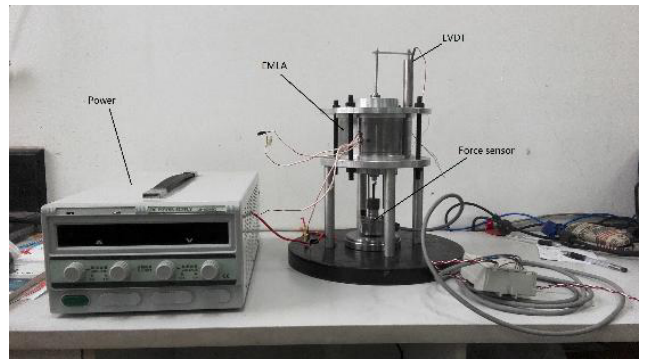

Figure 1.The test bed of EMLA

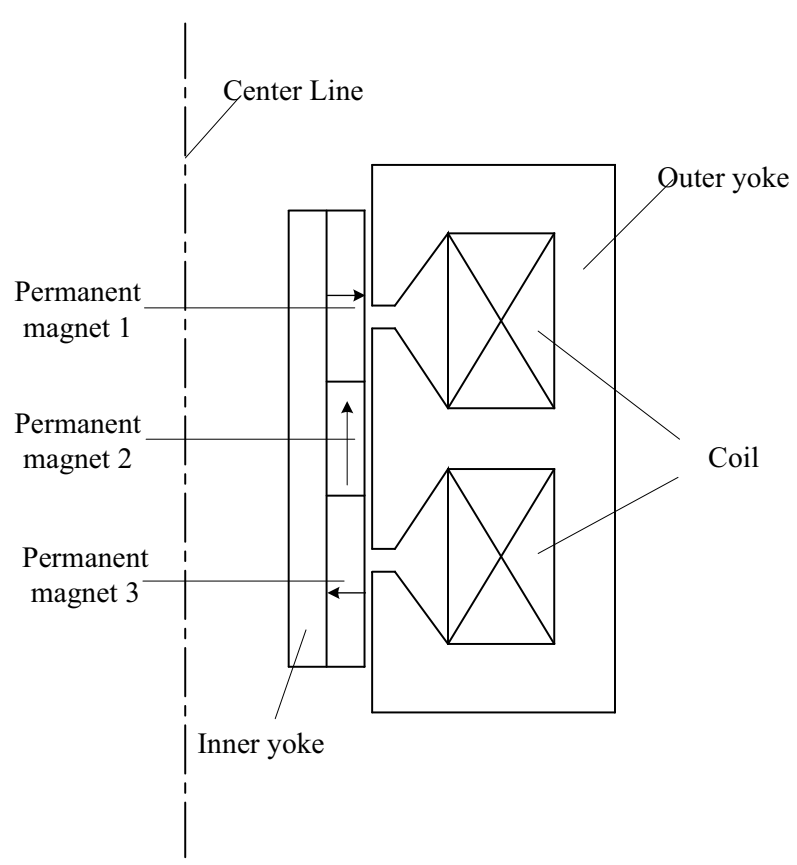

Figure 2.The structure of EMLA

Table 1. EMLA's performance parameters

\begin{tabular}{|c|c|}
\hline Parameters & value \\
\hline Resistance, $R(\Omega)$ & 5.4 \\
\hline Inductance, $L(\mathrm{mH})$ & 20 \\
\hline Motor Constant, $K_{i}(\mathrm{~N} / \mathrm{A})$ & 89 \\
\hline Stroke, $S(\mathrm{~mm})$ & \pm 25 \\
\hline Maximum Thrust, $F_{\text {peak }}(\mathrm{N})$ & 1000 \\
\hline
\end{tabular}

\section{$2.21 / 4$ electromagnetic active suspension system modeling}

By replacing the original damper, quarter electromagnetic active suspension with single degree of freedom which are composed of vehicle body mass and EMLA paralleledwith a spring is modeled (Fig.3). Based on Newton's second law, the dynamic equation is:

$$
m \ddot{z}+C(\dot{z}-\dot{q})+K(z-q)=0
$$

where $m$ is the sprung mass, $k$ is the spring stiffness, $C$ is the damping coefficient of the EMLA, $q$ is the road roughness function, and $z$ is the vertical displacement of the sprung mass[4].

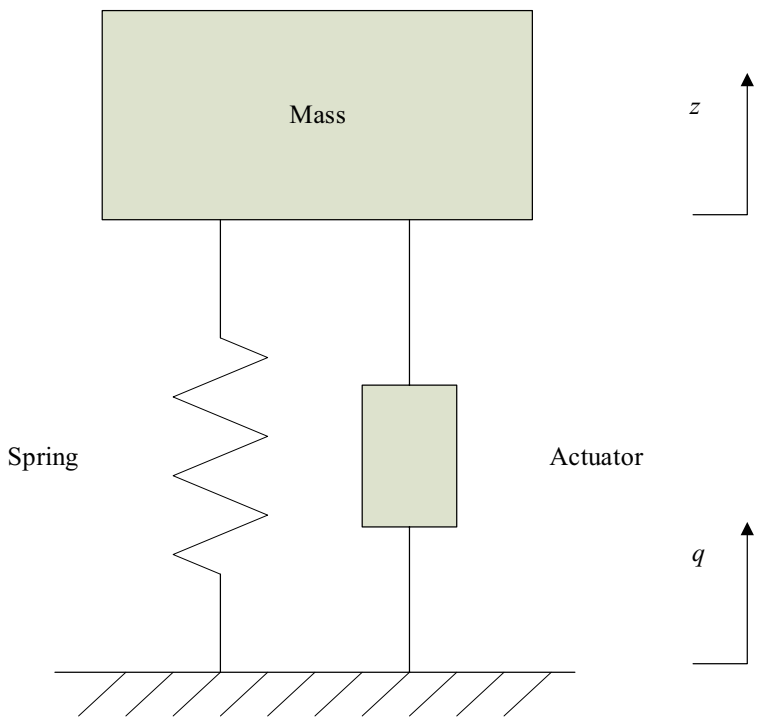

Figure 3.1/4 electromagnetic active suspension model

\section{Co-simulationwith maxwell and simplorer}

The characteristics of the EMLA in generator mode under variable sinusoidal excitation are accurately investigated by using a co-simulation between Maxwell and Simplorer. Maxwell is an interactive software package that uses finite element analysis (FEA) to solve three-dimensional (3D) electrostatic, magneto static, eddy current and transient problems. Simplorer is an integrated, multidomain, mixed-signal simulator for complex technical systems [5]. The EMLA is established in Maxwell firstly by setting the initial parameters such as the structure size, material properties and coils, etc. Then, the quarter electromagnetic active suspension is set up in Simplorer, including the mechanical part and electronic part which is shown in Fig.4. The EMLA in Maxwell has been previously loaded into Simplorer through the Sub circuit. The EMLA model has 4 pins, namely 'Winding in', 'Winding_out', 'Motion Setup_in', and 'Motion Setup_out', respectively. Road vibration is given by position excitation through pin 'Motion Setup in'. When a Transient Co simulation Component circuit is used in a Simplorer design, Maxwell 2D or 3D transient and Simplorer transient solvers exchange data during each simulation time step. Maxwell uses a loop form of the circuit equations, while Simplorer uses a nodal form of the circuit equations. After each time step, Simplorer forms a Norton-equivalent of the drive circuit at the coupling pins between the Maxwell component and the rest of the system. Maxwell converts this to a loop matrix, solves the finite element equations, and then outputs a Thevenin-equivalent for the next Simplorer time step. Simplorer controls the time step during simulation, and also controls the linear velocity of the Maxwell 
component. This parameter-based coupling enhances solution accuracy and stability.

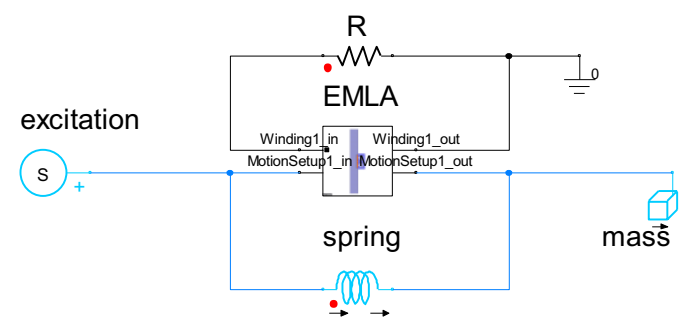

Figure 4.1/4 electromagnetic active suspension model in Simplorer

\section{Results analysis}

In order to observe the characteristic of the EMLAconveniently, the road disturbances are assumed as sinusoidal displacement excitation, the mechanical power, electric power, and efficiency $\eta$, defined as the ratio of electric energy to mechanical energy are given by the formulas as follows:

$$
\begin{aligned}
& P_{m}=\frac{1}{t} \int_{0}^{t} F v d t \\
& P_{e}=\frac{1}{t} \int_{0}^{t} u i d t \\
& \eta=\frac{P_{m}}{P_{e}} * 100 \%
\end{aligned}
$$

where $t$ is the simulation time, $F$ is the EMLA's thrust force, $v$ is moving speed of mover of the EMLA , $u$ and $i$ are voltage and current of the coils, respectively.

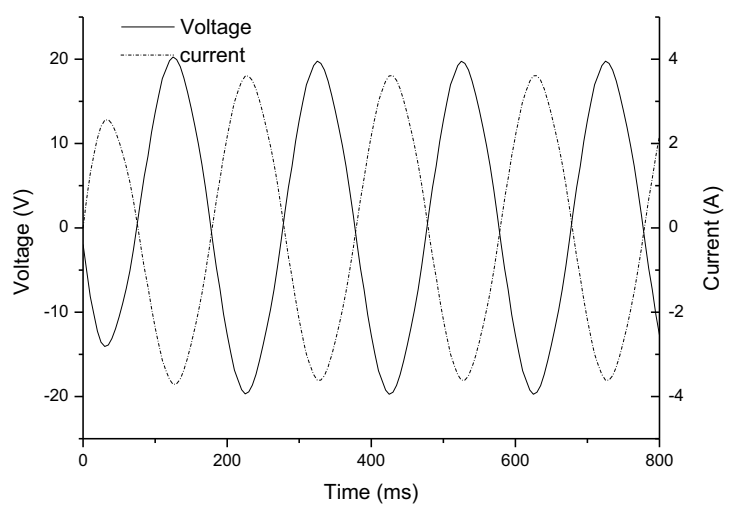

Figure 5.The voltage and current of EMLA

As shown in Fig. 5 and Fig.6, phase difference of $180^{\circ}$ exists between voltage and the current, and this phenomenon also occurs between the force and the speed. Thus, mechanical power and electronic power are negative, which means the actuator is in generator mode. Fig.7 and Fig. 8 show that the absolute value of the average of mechanical power and electric power are both proportional to the frequency and the amplitude of excitation. Fig.9 shows that the efficiency is inversely proportional to the frequency and changes nonlinearly with the increase of stroke. The higher efficiencies always occur in the stroke from $6 \mathrm{~mm}$ to $9 \mathrm{~mm}$ no matter how the frequency changes, and the maximum is up to $94 \%$ at the frequency of $2 \mathrm{~Hz}$.

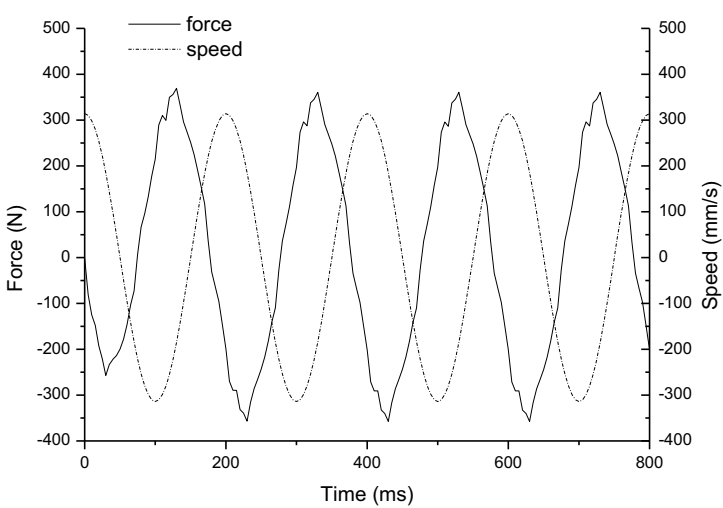

Figure 6. The force and speed of EMLA

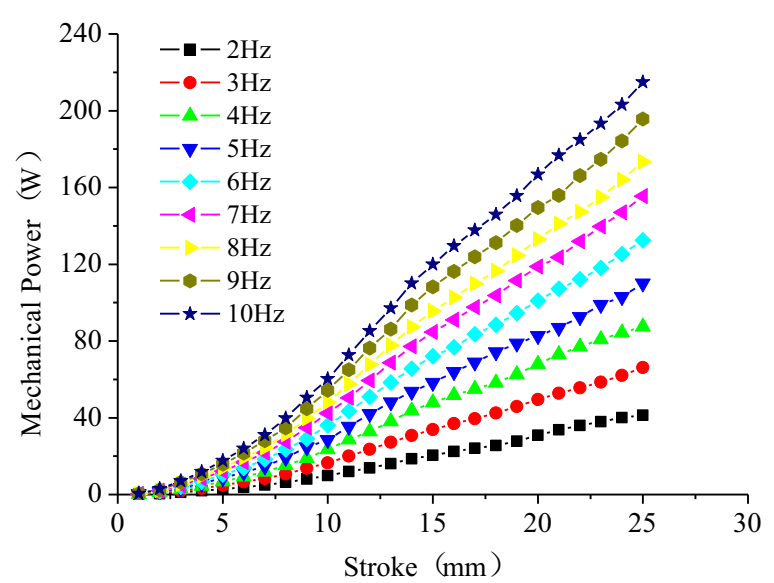

Figure 7. The absolute value of the average of mechanical power under different excitations

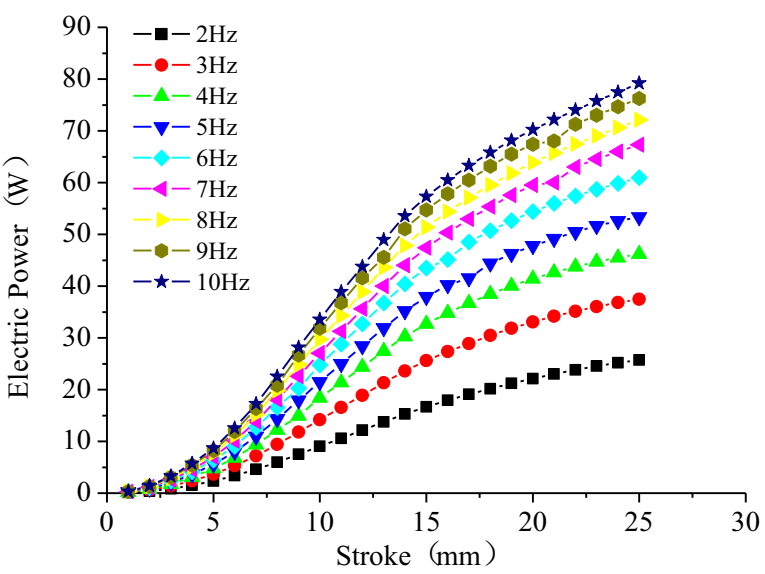

Figure 8. The absolute value of the average of electric power under different excitations 


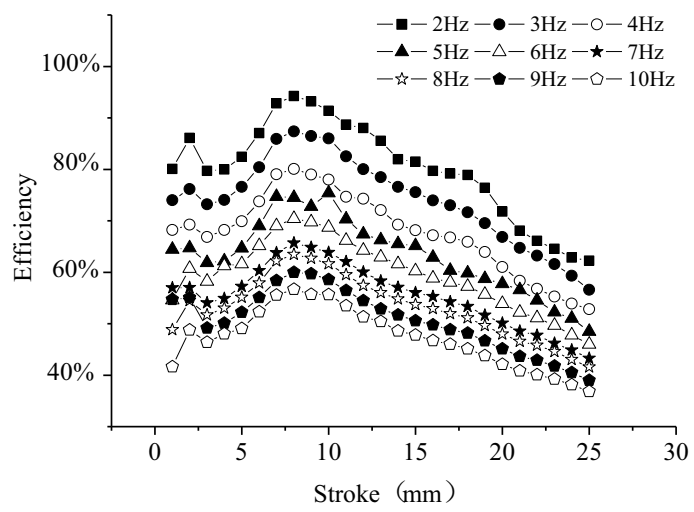

Figure 9. Efficiency of EMLA under different excitations

\section{Conclusion}

The EMLA, added into the active suspensions, has the benefits of altering vibration energy into electric energy while with the feature of damping road disturbances. It will first be of importance to analyze the power generation characteristics of the EMLA under different excitations. Maxwell and Simplorer co-simulation is proposed as a method to investigate how the mechanical power and the electric power change along with the excitations. The simulations show that the mechanical power and electric power are proportional to the frequency and the amplitude of excitation. The ratio of electric energy to mechanical energyvaries inversely with the increase of frequency and changes nonlinearly with the increase of stroke.

\section{Acknowledgment}

This work was supported by the Natural Science Foundation of Jiangsu Province (Grant No. BK20130757) and the Peak of the Six Talents of Jiangsu Province (Grant No. 2015-JXQC-004).

\section{References}

1. B.L.J. Gysen, J.J.H. Paulides, J.L.G.Janssen, E.A.Lomonova, IEEE. T. VEH. TECHNOL., 59, 3 (2010)

2. J.B. Wang, W.Y Wang, K. Atallah. IEEE. T. VEH. TECHNOL.,60, 1 (2011)

3. M.Morteza, S. Mehdi, H. Sahar,IEEE T IND ELECTRON., 60, 10 (2013)

4. I.Martins, J.Esteves, G.D.Marques, F. Pina da. Silva, IEEE. T. VEH. TECHNOL., 55, 1(2006)

5. J.H.Yu,B.D.Bai, D.Z.Chen, Z.F. Hu,WCICA, 11th, 2015. 